\title{
VIOLENCIA FAMILIAR: PROGRAMAS DE ATENCIÓN PARA HOMBRES QUE EJERCEN VIOLENCIA (HEV)
}

\section{Domestic violence: Care programs for men who engage in violence (MEV)}

\author{
Sabina Deza Villanueva*
}

\begin{abstract}
Resumen
Se presenta el panorama actual de las intervenciones psicosociales con varones, dedicadas a la erradicación de la violencia contra las mujeres, describiéndose las particularidades de los programas de atención para hombres que ejercen violencia que se realizan tanto en los países desarrollados como en los del tercer mundo, desde hace más de treinta años. Así mismo se explicitan las diferentes tipologías de atención que van desde las estrategias de promoción de la no violencia, la prevención primaria, secundaria y terciaria en sus diversas formas, hasta las de fomento del compromiso activo de los no violentos en acciones para erradicar la violencia de género.
\end{abstract}

Palabras clave: violencia familiar, hombres que ejercen violencia, programas de atención.

\begin{abstract}
We introduce the current picture of psychosocial interventions with men devoted to eradicating violence against women, describing the special care program for men who exercise violence taking place in both the developed and the Third World countries for over thirty years. It also makes clear the different types of care ranging from strategies to promote non-violence, primary, secondary and tertiary prevention in its various forms, to the promotion of active involvement of non-violent people in actions to eradicate gender violence.
\end{abstract}

Keywords: Family violence, men who engaged in violence, care programs.

\footnotetext{
* Psicóloga. Profesora de la Facultad de Psicología y Humanidades de la Universidad Femenina del Sagrado Corazón, UNIFÉ. Consultora del Programa Nacional contra la Violencia Familiar y Sexual, del Ministerio de la Mujer y Desarrollo Social (PNCVES-MINDES). sabinadeza@terra.com.pe
} 
La violencia familiar constituye un abuso contra los derechos humanos y uno de los problemas de salud pública fundamentales y crecientes en todo el mundo. $\mathrm{Al}$ mismo tiempo es una manifestación extrema de la desigualdad de género que afecta a las mujeres, según el "Informe mundial sobre la violencia y la salud", de la Organización Panamericana de la Salud (2002).

La violencia del compañero íntimo es un problema que afecta y pone en riesgo a la salud de las mujeres. Se ha estimado que entre $13 \%$ y $61 \%$ de las mujeres ha sufrido algún tipo de maltrato físico por parte de su pareja en algún momento de su vida (CarrascoPortiño, Vives-Cases, Gil-González y Álvarez-Dardet, 2007). Además, en la literatura científica se alude a un posible "efecto acumulativo" de los diversos tipos de maltrato, de manera que el impacto en la salud es mayor en las mujeres que experimentan abusos físicos, sexuales y psicológicos que en las que experimentan un solo tipo de abuso. La gravedad de este problema en la salud y en el bienestar de las mujeres se pone de manifiesto también en el impacto económico y social que conlleva (Ellsberg y Clavel-Arcas, 2001).

Las intervenciones psicosociales con varones dedicadas a la erradicación de la violencia contra las mujeres se realizan en diferentes países desde hace años, y son de diferentes tipos, desde las estrategias de promoción de la no violencia, la prevención primaria, secundaria y terciaria de sus diversas formas, hasta las de fomento del compromiso activo de los no violentos en acciones para erradicar dicha violencia (RamírezRodríguez, 2006).

La violencia de género es un problema en alza y adquiere actualmente unas cifras alarmantes. En el Perú, el feminicidio y la violencia de género se han convertido en una incomprensible realidad. Entre 2004 y 2007 murieron más de 400 mujeres a causa de asesinatos, crímenes de odio, violencia o incluso suicidios como resultado de maltratos y abusos. En al menos el 20 por ciento de los casos la mujer ya había denunciado a su agresor, cuyo perfil se puede definir como varón (93 por ciento), entre 26 y 45 años (49 por ciento), pareja sentimental de la víctima (45 por ciento) o ex pareja (20 por ciento) manifestantes de celos descontrolados (34 por ciento), sospecha de infidelidad y con antecedentes de maltrato a la pareja. La premeditación del crimen se dio en uno por cada cuatro casos de feminicidio (Ministerio de la Mujer y Desarrollo Social/Programa Nacional contra la Violencia Familiar y Sexual [MIMDES/PNCVFS], 2007). Estas cifras alarmantes que se repiten a nivel mundial (Russel y Harmes, 2006), han provocado en la comunidad científica un aumento del interés por el estudio de los agresores.

Desde una perspectiva clínica, diversos autores han tratado de identificar las características de los hombres violentos contra la mujer; hallando que los agresores suelen presentar carencias psicológicas significativas, como sesgos cognitivos -pensamientos distorsionados sobre los roles sexuales y la inferioridad de la mujer, principalmente-, dificultades de comunicación, irritabilidad y una falta de control de los impulsos, así como otras dificultades específicas (abuso de alcohol y celos patológicos) (Dutton y Golant, 1999; Fernández-Montalvo y Echeburúa, 1997; citados por Echeburúa, Corral, Fernández- Montalvo y Amor, 2004). Hubo un esfuerzo considerable por identificar tipos de agresores, pero todavía se carecen de datos empíricos sólidos en apoyo de una clasificación. Sin embargo, coincidiendo, con Echeburúa et al. (2004), Madina (2005) afirma que los maltratadores suelen ser personas con dificultades para controlar sus impulsos, machistas, dependientes emocionalmente, con dificultad para expresar emociones, generalmente descargan su agresividad en el hogar, tienen celos irracionales, entre otras características.

Entre los trastornos de personalidad frecuentemente encontrados en los agresores, han sido el trastorno antisocial de la personalidad, el límite y el narcisista (Hamberger \& Hastings, 1988).

Desde una perspectiva terapéutica, se ha observado también un interés creciente por el tratamiento psicológico de los agresores, sobre todo en un medio comunitario. De este modo, han surgido algunos programas específicos de intervención con este tipo de agresores. Los resultados obtenidos en alguno de ellos -especialmente con los hombres que completan totalmente el programa de intervención-son controversiales, por un lado tenemos los que reportan cambios positivos, reconociendo que una dificultad existente es la negación -o, al menos, la minimización del problema- por parte del agresor, y por otro lado, los que atribuyen a la pareja el origen y mantenimiento del conflicto, lo que puede llevar a un rechazo del tratamiento o a un abandono prematuro del mismo. 
De otra parte, los cuestionamientos surgen a partir de la consideración que algunos de estos programas están sobredimensionando sus resultados y creando falsas expectativas sobre su eficacia a partir de evaluaciones nada rigurosas hechas en plazos irrelevantes y con un número ínfimo de varones estudiados (Bonino, 2005).

Para lograr resultados fiables, la experiencia internacional indica que estos programas deben ser específicos, alejados de la concepción del "maltratador" como "enfermo" y de una inespecificidad "reeducativa" o de "control de la ira". Especificidad significa en primer lugar tener en cuenta la particularidad de la violencia masculina contra la mujer. Desde la perspectiva de género, esta violencia está basada en un sistema de creencias sexista y en los mandatos de la masculinidad hegemónica (Bonino, 2000), que promueven expectativas de dominio, emociones y comportamientos que naturalizan su uso. Creencias, que por otra parte son la manifestación en lo individual de una cultura que legitima la violencia y que aún sigue dando a los varones más derechos. Con esta base, la violencia contra las mujeres está asentada en motivaciones de control y no en motivaciones "afectivas" aunque éstas puedan potenciarla. Consiste, en una "acción continuada" -no en una "reacción agresiva"-, una estrategia de control variada y frecuentemente sistemática que los varones que la ejercen utilizan desde un esquema mental machista y misógino-, para impedir la libertad y autonomía de la mujer y llevarla a hacer lo que ellos quieren. Esta estrategia se transforma frecuentemente en un modus vivendi abusivo que puede incluir la agresividad manifiesta y el "desborde" emocional.

Los modos de pensar, sentir y actuar de hombres y mujeres llevan sobre sí el peso indiscutible de la cultura en los diferentes momentos del desarrollo. El mantenimiento del poder en el hombre desde lo simbólico, desde lo que la sociedad y la cultura han establecido, incorporando en su proceso de socialización un conjunto de valores, creencias y actitudes que en su configuración más estereotipada delimitan la denominada mística masculina: restricción emocional, homofobia, modelos de control, poder y competencia. Al respecto existe todo un grupo de mitos y creencias construidas y compartidas que dan sustento a tal socialización masculina (Corsi, 1999); por ejemplo:
- La masculinidad es la forma más valorada de la identidad genérica;

- La vulnerabilidad, los sentimientos y las emociones en el hombre son signos de feminidad y deben ser evitados;

- El autocontrol y el control sobre los otros y sobre su entorno son esenciales para que el hombre se sienta seguro;

- El éxito masculino en las relaciones con las mujeres está asociado a la subordinación de la mujer a través del uso del poder y el control de la relación;

- La sexualidad es el principal medio para probar la masculinidad.

De otro lado, la especificidad de estos programas significa también que en ellos deben utilizarse herramientas diagnósticas, psicoterapéuticas y educativo-cuestionadoras apropiadas para intentar lograr promover el cambio de la estrategia del maltratador hacia formatos no violentos e igualitarios. Un trabajo de este tipo es un asunto delicado que requiere calibrar el riesgo de incidentes futuros de violencia, una evaluación integral individualizada y conocer los resortes terapéuticos de la promoción del cambio sabiendo sobre qué procesos emocionales, cognitivos y motivacionales hay que incidir, cómo modificar el esquema mental, las creencias que sustentan la violencia, y las resistencias al cambio, y con qué instrumentos (Corsi, 1999). Con éstos se debe intentar lograr que los varones no sólo detengan su comportamiento, sino que examinen las emociones con las que complacientemente justifican su actuación, y fundamentalmente que se pregunten sobre sus motivaciones, en especial por qué se sienten con derecho a maltratar a la mujer. Y a partir de ahí construyan nuevas vías de relación con las mujeres, otras formas de gestión de los conflictos y nuevos modos de hacerse cargo de sus emociones (Corsi, 2000).

La motivación para el tratamiento es el motor del cambio y la piedra angular del éxito en un programa terapéutico con maltratadores. No se trata tan sólo de la motivación inicial para acudir a las sesiones, sino de la motivación necesaria para mantenerse en el programa y cumplir adecuadamente las prescripciones terapéuticas. 
Dado lo específico de este tipo de intervenciones, quienes la lleven a cabo deben ser personas específicamente formadas para ello. Formarse para el trabajo con maltratadores requiere aprender nuevos instrumentos pero sobre todo desaprender un modo tradicional de pensar, sentir y tratar la cuestión, junto a un trabajo personal de revisión de las propias creencias genéricas.

Los programas para varones que ejercen violencia contrala mujer con la que se tiene o ha tenido vinculación afectiva (pareja o ex pareja), existen en el mundo anglosajón y escandinavo (EEUU, Canadá, Suecia, Australia) desde hace treinta años, y desde hace quince en el de habla hispana (Argentina, México) (Corsi, 1999). En estos últimos se los denomina habitualmente "programas de intervención con maltratadores (o agresores)", o "programas para varones que renuncian a la violencia". Se crearon como parte de las acciones de reinserción social y de prevención de reincidencias de dichos varones y se han planteado habitualmente como un complemento a las intervenciones con las mujeres. Ofrecen servicios a varones que acuden derivados desde los sistemas sanitario, de servicios sociales, judicial (la vía de acceso más frecuente), o los menos, por iniciativa propia.

Desde el punto de vista de la denominación, es claro que estos programas no son "tratamientos tradicionales" en tanto los varones que ejercen violencia no son enfermos, ni estrictamente son de "rehabilitación" (volver a habilitar) ya que justamente han carecido de la habilidad de la no violencia. Lo que sí puede pensarse es en contribuir a que cambien renunciando a la violencia y al abuso, "educándolos" y "habilitándolos" para comportamientos igualitarios y pacíficos a través de estrategias psicoeducativas y así "recuperarlos" para la sociedad (Bonino, 2005). La experiencia acumulada indica que es fundamental que sus condiciones de aplicación sean coherentes con la naturaleza injusta, dañina, atentatoria contra los derechos humanos y delictivos de la violencia. Por ello, en la mayoría de los países antedichos, los programas priorizan la seguridad de la víctima, y cuando hay condena judicial no se plantean como alternativa a ella.

A diferencia del término "hombres violentos" que etiqueta y cataloga el problema como una cuestión del "ser masculino", de identidad y, por tanto aparentemente ajeno a una responsabilidad propia, el término "hombres que ejercen violencia" HEVsupone una propuesta en la que se incluye la violencia como un proceso aprendido, no de la identidad, sino del "hacer", del comportamiento que como tal podría no ejercerse y que, además, supone responsabilidad y reaprendizaje (Cerezo, 2004).

Para asegurar que los programas para varones que ejercen violencia en la pareja sean efectivos y no se vuelvan en contra de las mujeres, la intervención del Estado en cuanto a su control de calidad es fundamental. En muchos países anglosajones este control se realiza desde hace muchos años a través del diseño de requisitos mínimos que indican objetivos y requerimiento profesionales y técnicos necesarios, y que son de obligado cumplimiento para que un programa se ofrezca como servicio público (Bonino, 2000).

La intervención con HEV aunque comparte los principales principios de la práctica clínica general, específicamente de las psicoterapias (individual y grupal), no puede limitarse al enfoque clínico general, ya que el problema de la violencia hacia las mujeres debe entenderse en el contexto de los procesos de construcción social de los géneros y la distribución desigual del poder en la sociedad patriarcal, y no únicamente como aspectos derivados de desórdenes de personalidad o de psicopatología individual (Campos, 2005). El enfoque de la psicopatología, aunque aporta elementos muy importantes para entender el comportamiento del hombre agresor, requiere de la comprensión de ese contexto sociocultural y político (Arce y Fariña, 2006). Y precisamente ese contexto tiene que ver con los procesos de socialización de género y la construcción de la identidad masculina, con los encargos, mandatos y exigencias que se hacen a los hombres (Velásquez, 2003).

La intervención con los $\mathrm{HEV}$ es un proceso resocializador, en el sentido de que es una experiencia de contención y a la vez una experiencia correctiva, que posibilita la comprensión, análisis y modificación de los patrones básicos y pautas de interacción de los hombres con las personas significativamente importantes para ellos (Ruiz-Jarabo y Blanco Prieto, 2004).

La intervención supone el trabajo simultáneo en varios niveles de análisis y de abordaje (cognitivo- 
ideológico, emocional-afectivo y el comportamental). El nivel cognitivo se refiere a los sistemas de pensamiento, creencias, ideas, con los cuales los hombres interpretan y perciben el mundo que los rodea. Las cogniciones se relacionan de manera directa con los sentimientos y las acciones, de tal forma que un hombre que posee un sistema de pensamientos y cogniciones propios de la masculinidad de orientación patriarcal, es probable que reprima sus sentimientos más profundos y que actúe de manera violenta ante las frustraciones.

El nivel cognitivo constituye un marco de referencia básico para entender, valorar e interpretar sus propias acciones y las de sus parejas. Este aspecto cognitivo tiene un papel muy importante en las emociones que estos hombres experimentan y en su respectivo manejo práctico. También es prioritario trabajar el aspecto ideológico, que es más que el cognitivo. El aspecto ideológico tiene que ver con el universo simbólico, propio de la cultura en la que los hombres han sido socializados y viven. La ideología patriarcal sustentada en principios religiosos, filosóficos, políticos es incorporada por los hombres no solamente a través de códigos o mensajes verbales, sino fundamentalmente a partir de una base emocional por medio de imágenes, íconos, signos, símbolos, significantes, los cuales se tornan muchas veces inconscientes y conforman parte del mundo interno de estos hombres.

En el nivel ideológico tenemos la justificación y defensa que se hace de las instituciones propias del patriarcado (misoginia, falocentrismo, androcentrismo, homofobia). El aspecto ideológico se ubica como el aspecto más de fondo, y tiene que ver con la posibilidad de estar convencido o no, de creer o no, con una forma de actuación pacifista, y que implica que el sujeto haga rupturas con sus anteriores sistemas de pensamiento. Aunque un hombre haya identificado algunas de sus distorsiones cognitivas o creencias irracionales respecto a su pareja y conozca algunas de las técnicas de reestructuración cognitiva, esto no garantiza que haya modificado los aspectos ideológicos de fondo, que tienen que ver con el aspecto más volitivo y con el querer mantener relaciones armoniosas en sus relaciones de pareja y familia.

Además de trabajar los aspectos cognitivoideológicos, es necesario trabajar con el nivel de las emociones y afectos en varios sentidos. Por un lado, el reconocimiento, identificación, expresión y validación de las emociones y sentimientos es uno de los propósitos fundamentales. De hecho, los hombres presentan dificultades en las áreas mencionadas. Se les hace muy difícil identificar, reconocer, expresar y validar sus emociones, especialmente si éstas tienen que ver con sentimientos de dolor, pérdida, temor, inseguridad. Tales emociones tienden a ser transformadas en enojo e ira. Las emociones se convierten en un problema para los hombres si están relacionadas con su vulnerabilidad.

En este sentido, las ansiedades y fantasías de abandono, pérdida, fragilidad, rechazo, entre otras, vulnerabilizan mucho a los hombres y tienden a evitar hablar de estas experiencias, o cuando lo hacen, lo hacen de una manera distorsionada, evitando tomar contacto con la carga emocional y con el sufrimiento. Prefieren actuarlo a través de conductas violentas que elaborarlo de manera constructiva. De la misma manera, los hombres tienden a racionalizar en torno a sus emociones, prefieren pensar acerca de lo que sienten que expresar sus sentimientos. Experimentar vulnerabilidad también es asociado con lo femenino y con ser mujer, y en la constitución de la masculinidad es fundamental demostrar que no se es mujer alejándose de todo aquello que se asemeje a eso (Fernández, 2005).

El problema de los hombres que ejercen violencia -HEV-, es un problema que supera lo psicológico para enmarcarse en la esfera más amplia de lo social. Por ello, las intervenciones psicoeducativas no deben ofrecerse como solución a la violencia sino como un componente más de un abordaje que ha de ser multidisciplinario y que debe implicar una respuesta comunitaria coordinada. En este sentido, no debemos perder de vista soluciones que caigan fuera del campo de la psicoterapia, como la condena por los delitos cometidos o la educación en valores no sexistas (Muñiz y Lopo, 2004). ¿Cuáles son entonces las razones que llevan a incluir la intervención psicoeducativa de los HEV dentro de un enfoque integral de la violencia familiar?

Las intervenciones con los hombres que ejercen violencia contra su pareja se justifican socialmente por la oportunidad que hay que dar a los agresores para cambiar su conducta (al margen de la necesidad de hacer frente a sus responsabilidades), por la 
protección a las víctimas actuales (muchas de ellas siguen conviviendo con el agresor), por la prevención de la violencia con posibles víctimas futuras y por la evitación de la extensión de la violencia a los hijos. Se trata además de interrumpir la cadena de transmisión intergeneracional y el aprendizaje observacional por parte de los hijos (Echeburúa et al., 2004; Sarasua y Zubizarreta, 2000). La intervención con los HEV se hace aún más necesaria cuando el riesgo de violencia grave contra la pareja es alto (Andrés-Pueyo y Redondo, 2007). Para evaluar este riesgo se cuenta actualmente con instrumentos de evaluación, como el Spousal Assault Risk Assessment Guide (SARA) (Kropp, Hart, Webster \& Eaves, 1994, 1995, 1998, citados por Kropp \& Hart, 2000).

Pero además, se ha observado que el enfoque judicial de los HEV suele ser insuficiente, y los resultados de los estudios indican una reducción en el uso directo de la violencia física en aquellos hombres que participan en los programas de tratamiento para maltratadores (Gerlock, 1997, citado por Espantoso, 2004).

A nivel de tratamiento, la falta de reconocimiento del problema o la adopción de una actitud soberbia de autosuficiencia, con un aparente dominio de la situación, dificultan la intervención. Es preciso evaluar en el primer contacto con el HEV el nivel de motivación para el cambio, así como su grado de peligrosidad actual, porque la protección de la víctima resulta prioritaria en este contexto (Sarasua, y Zubizarreta, 2000). En general la motivación inicial para el tratamiento en el HEV suele ser débil e inestable, sobre todo en aquellos que acuden al tratamiento por vía judicial. La decisión genuina de acudir a un programa psicoeducativo o terapéutico se adopta sólo cuando se dan varios requisitos previos: reconocer que existe un problema y que, además, causa sufrimiento a otras personas; darse cuenta de que el sujeto no lo puede resolver por sí solo; y, por último, valorar que el posible cambio va a mejorar el nivel de bienestar actual. El hombre violento va a estar realmente motivado cuando llega a percatarse de que los inconvenientes de seguir maltratando superan a las ventajas de no hacerlo (Echeburúa et al., 2004).

Los programas de tratamiento para HEV comienzan a desarrollarse a mediados de los años 70, y desde entonces han ido evolucionando en función de los distintos modelos explicativos en los que se han ido sustentando. Así, entre las primeras explicaciones que se dan al fenómeno de la violencia doméstica está la psicopatológica (Dutton, 1997). En un primer momento, los investigadores empezaron a acercarse a la problemática del hombre violento considerándolo psicológicamente enfermo y, por lo tanto, priorizaron identificar las patologías que estaban en la base de su personalidad. Una de las definiciones más frecuentes que se dieron fue que los hombres violentos poseían una personalidad sádica o bien pasivo-agresiva, que eran individuos con características paranoides, personalidades límite, etc.; en definitiva, encuadrables dentro de un síndrome psiquiátrico.

También hubo intentos de explicar la conducta del hombre violento adscribiéndola a un modelo más amplio de explicación de la conducta agresiva apoyado en elementos de tipo biológico o hereditario. La hipótesis es que el funcionamiento biológico del hombre es diferente del de la mujer y por lo tanto, está más predispuesto a las conductas violentas.

Un tercer tipo de explicaciones proviene de los enfoques sociológicos y, en términos generales, sostiene que la violencia conyugal es una de las formas que adopta la dominación del hombre sobre la mujer, en el marco de una sociedad patriarcal. La agresión a las mujeres, por lo tanto, es una forma de violencia "normal"; los que la cometen no son enfermos mentales si no personas que creen que ejercer el patriarcado es su derecho, que el matrimonio les confiere la facultad irrestricta de controlar a sus esposas y que el castigo físico es un medio aceptable de establecer ese control. En realidad, la violencia mantiene el statu quo, con los hombres en la cima de la jerarquía del poder.

Finalmente, otro grupo de teorías intenta explicar la conducta del hombre violento desde un punto de vista más estrictamente psicológico. Aquí encontramos aquellas teorías que afirman que la conducta violenta del hombre dentro de su hogar es aprendida, y que está en relación con las experiencias vividas durante su infancia (Corsi, 1995). En concreto, la observación reiterada por parte de los hijos de la violencia ejercida por el hombre a la mujer tiende a perpetuar esta conducta en las parejas de la siguiente generación. Los niños aprenden que la violencia es un recurso eficaz y aceptable para hacer frente a las frustraciones del hogar. Y las niñas, a su vez, aprenden que ellas deben aceptarla y convivir con ella (Echeburúa y Corral, 1998). 
En líneas generales, estos son los modelos que históricamente han guiado la investigación y el desarrollo de los programas de tratamiento para hombres violentos. De este modo, los primeros programas se basaban exclusivamente en estrategias para el control o manejo de la ira (como las técnicas de relajación, las autoinstrucciones, los autorregistros o la técnica de "tiempo fuera"). Con la inclusión de la perspectiva feminista se introducen las nociones de poder y género; y el modelo del aprendizaje social enfatiza el análisis de la violencia en la familia de origen. Ninguno de estos modelos está libre de críticas; y quizá la mejor forma de entender cada uno de ellos sea considerarlos como una aproximación parcial a la explicación de la violencia de género (Jacobson y Gottman, 2001). En este sentido, y como afirma Ravazzola (1997): "ante el problema de la violencia familiar, tan repetitivo y difícil de solucionar, parece más sensato sumar que restar, valorizar los aportes positivos, y revisar y modificar lo que no ayuda" (citada por Espantoso, 2004, p. 77)

En el Perú se han realizado experiencias de trabajo con hombres que ejercen violencia tomando como modelo la propuesta mexicana del Programa de Hombres que Renuncian a su Violencia, PHRSV, el cual forma parte de una iniciativa integral para escuchar, comprender e intervenir con hombres que ejercen violencia contra sus parejas e hijas/ os. Fue ejecutado en sus inicios por un equipo multidisciplinario, coordinado desde la Unidad de Sexualidad y Salud Reproductiva de la Facultad de Salud Pública y Administración de la Universidad Peruana Cayetano Heredia; y teniendo presencia en 04 distritos de Lima Metropolitana (Ramos, 2005). La idea de implementar este servicio surge a partir de los resultados del estudio multicéntrico realizado con la Organización Mundial de la Salud (OMS): "Violencia Sexual y Física Contra las Mujeres en el Perú" (20002001); en el que participó la Unidad de Salud Sexual y Reproductiva de la Universidad Peruana Cayetano Heredia; la misma que da soporte a la coordinación del PHRSV.

En el 2002 y 2003, la Fundación Ford apoyó un Proyecto para investigar la violencia conyugal desde el lado de los hombres y elaborar una propuesta para trabajar con hombres agresores en Perú. En esta iniciativa, se visitaron y compartieron experiencias con diferentes organizaciones que realizan programas dirigidos a hombres que ejercen violencia contra sus parejas en Estados Unidos, Canadá, Chile y México. Luego de evaluar los diferentes modelos de intervención que se habían conocido, se decidió que el desarrollado por el Colectivo de Hombres por las Relaciones Igualitarias A.C. (CORIAC) era el que tenía mayor potencial de réplica en el Perú. Actualmente este Programa se lleva a cabo en el Distrito de Jesús María bajo la conducción del sociólogo Miguel Ramos. Posteriormente el PNCVFS en convenio con ADRA Perú, desarrollan desde el año 2007 el Proyecto de Atención Integral a las Personas que ejercen violencia (CAI).

\section{REFERENCIAS}

Andrés-Pueyo, A. y Redondo, S. (2007). Predicción de la violencia: entre la peligrosidad y la valoración del riesgo de violencia. Papeles del Psicólogo, $28,157-173$.

Arce, R. y Fariña, F. (2006). Programa Galicia de reeducación para maltratadores de género. Anuario de Psicología Jurídica, 16, 41-64.

Bonino, L. (2000). Violencia de género y prevención. El problema de la violencia masculina. En C. Ruiz-Jarabo y P. Blanco (Comp.), La violencia contra las mujeres. Prevención y detección. Madrid: Díaz de Santos.

Bonino, L. (2005). Los programas de reeducación, reinserción o rehabilitación para varones que ejercen violencia contra las mujeres - Riesgos de su implementación con estrategias inadecuadas. Madrid: Centro de estudios de la Condición Masculina.

Campos, A. (2005). La psicoterapia con hombres que presentan problemas de poder y control. Costa Rica: Instituto WEM.

Carrasco-Portiño, M., Vives-Cases, C., Gil-González, D. y Álvarez-Dardet, C. (2007). ¿Qué sabemos sobre los hombres que maltratan a su pareja? Una revisión sistemática. Rev. Panam. Salud Pública, 22(1), 55-63.

Cerezo, H. (2004). ¿Hombres violentos versus hombres que ejercen violencia? México: Centro de Crisis Casa Amiga. 
Corsi, J. (1995). Violencia masculina en la pareja. Buenos Aires: Paidós.

Corsi, J. (1999). Violencia masculina en la pareja. Una aproximación al diagnóstico y a los modelos de intervención. Buenos Aires: Paidós.

Corsi, J. (2000). Modelos de intervención con hombres que ejercen violencia en la pareja. Buenos Aires: UBA.

Dutton, D. G. (1997). El golpeador. Buenos Aires: Paidós.

Echeburúa, E. y Corral, P. (1998). Manual de violencia familiar. Madrid: Siglo XXI.

Echeburúa, E., Corral, P., Fernández-Montalvo, J. y Amor, P.J. (2004). ¿Se puede y debe tratar psicológicamente a los hombres violentos contra la mujer? Papeles del Psicólogo, 25(88), 10-18.

Ellsberg, M. y Clavel-Arcas, C.(2001). Sistematización del proyecto OPS: Hacia un modelo integral de atención para la violencia intrafamiliar en Centroamérica. Washington, D.C.: OPS/OMS.

Espantoso, R. (2004). ¿Qué se puede hacer con los agresores de mujeres? Cuad. Psiquiatr. Comunitaria, 4(1), 75-86.

Fernández, I. (2005). Violencia contra las mujeres en la relación de pareja: una demanda de seguridad ciudadana. Persona y Sociedad (Universidad Alberto Hurtado), 19(1), 51-72.

Hamberger, L.K. \& Hastings, J.E. (1988). Skills training for treatment of spouse abusers: an outcome study. Journal of Family Violence, 3, 121-130.

Jacobson, N. y Gottman, J. (2001). Hombres que agreden a sus mujeres. Barcelona: Paidós.

Kropp, P. R. \& Hart, S. D. (2000). The spousal assault risk assessment (SARA) guide: Reliability and validity in adult male offenders. Law and Human Behavior, 24(1), 101-118.
Madina, J. (2005). Perfil psicológico e intervención de los hombres maltratadores. Conferencia. Donostia-San Sebastián: Diputación Foral de Gipuzkoa. Departamento para los Derechos Humanos, el Empleo y la Inserción.

Ministerio de la Mujer y Desarrollo Social/Programa Nacional contra la Violencia Familiar y Sexual (MIMDES/PNCVFS). (2007). Feminicidio en el Perú. Lima. Recuperado de Estadísticas/ PNCVFS wWw.mimdes.gob.pe/estadisticas-pncvfs.html

Muñiz Torrado, J. A. y Lopo Lago, M. (2004). Procedimientos terapéuticos con hombres que agreden a sus mujeres. Cuad. Psiquiatr. Comunitaria, 4(1), 65-73.

Organización Panamericana de la Salud, Oficina Regional para las Américas de la Organización Mundial de la Salud. (2002). Informe mundial sobre la violencia y la salud: resumen. Washington, D.C.: Autor.

Ramírez-Rodríguez, JC. (2006). La violencia de varones contra sus parejas heterosexuales: realidades y desafíos. Un recuento de la producción mexicana. Salud Pública Mex, 48, Supl. 2, S315-S327.

Ramos, Miguel. (2005). Masculinidades y violencia. Un problema de hombres y mujeres. Perú: Universidad Peruana Cayetano Heredia.

Ravazzola, M. C. (1997). Historias infames: los maltratos en las relaciones. Buenos Aires: Paidós.

Ruiz-Jarabo, C. y Blanco Prieto, P. (Dirs.) (2004). La violencia contra las mujeres. Prevención y detección. Madrid: Díaz de Santos.

Russel, D. y Harmes, R. (2006). Feminicidio. Una perspectiva global. México: UNAM.

Sarasua, B. y Zubizarreta, I. (2000). Violencia en la pareja. Málaga: Aljibe.

Velásquez, S. (2003). Violencias cotidianas, violencia de género. Buenos Aires: Paidós. 